\title{
Testing the effectiveness and the contribution of experimental supercharge (reversed) end-to-side nerve transfer
}

\author{
*Mustafa Nadi, MD, Sudheesh Ramachandran, MCh, Abir Islam, Joanne Forden, Gui Fang Guo, \\ and Rajiv Midha, MD
}

Department of Clinical Neurosciences and Hotchkiss Brain Institute, Cumming School of Medicine, University of Calgary, Alberta, Canada

\begin{abstract}
OBJECTIVE Supercharge end-to-side (SETS) transfer, also referred to as reverse end-to-side transfer, distal to severe nerve compression neuropathy or in-continuity nerve injury is gaining clinical popularity despite questions about its effectiveness. Here, the authors examined SETS distal to experimental neuroma in-continuity (NIC) injuries for efficacy in enhancing neuronal regeneration and functional outcome, and, for the first time, they definitively evaluated the degree of contribution of the native and donor motor neuron pools.
\end{abstract}

METHODS This study was conducted in 2 phases. In phase I, rats $(n=35)$ were assigned to one of 5 groups for unilateral sciatic nerve surgeries: group 1, tibial NIC with distal peroneal-tibial SETS; group 2, tibial NIC without SETS; group 3, intact tibial and severed peroneal nerves; group 4, tibial transection with SETS; and group 5, severed tibial and peroneal nerves. Recovery was evaluated biweekly using electrophysiology and locomotion tasks. At the phase I end point, after retrograde labeling, the spinal cords were analyzed to assess the degree of neuronal regeneration. In phase II, 20 new animals underwent primary retrograde labeling of the tibial nerve, following which they were assigned to one of the following 3 groups: group 1, group 2, and group 4. Then, secondary retrograde labeling from the tibial nerve was performed at the study end point to quantify the native versus donor regenerated neuronal pool.

RESULTS In phase I studies, a significantly increased neuronal regeneration in group 1 (SETS) compared with all other groups was observed, but with modest (nonsignificant) improvement in electrophysiological and behavioral outcomes. In phase II experiments, the authors discovered that secondary labeling in group 1 was predominantly contributed from the donor (peroneal) pool. Double-labeling counts were dramatically higher in group 2 than in group 1, suggestive of hampered regeneration from the native tibial motor neuron pool across the NIC segment in the presence of SETS.

CONCLUSIONS SETS is indeed an effective strategy to enhance axonal regeneration, which is mainly contributed by the donor neuronal pool. Moreover, the presence of a distal SETS coaptation appears to negatively influence neuronal regeneration across the NIC segment. The clinical significance is that SETS should only employ synergistic donors, as the use of antagonistic donors can downgrade recovery.

https://thejns.org/doi/abs/10.3171/2017.12.JNS171570

KEYWORDS nerve injury; nerve transfers; rat; retrograde labeling; sciatic nerve; peripheral nerve

0 EVERE peripheral nerve injuries represent one of the leading causes of lifelong disability, affecting 3\% of trauma patients and often resulting in poor functional recovery. ${ }^{32}$ Severe neuroma in-continuity/nerve incontinuity (NIC) injuries are difficult to manage clinically because of their occult nature, together with poor functional outcome due to misdirected axon regeneration and

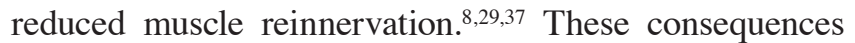
are even more deleterious in cases of proximal NIC, where the time to reinnervation is substantially high. ${ }^{12,13}$ Therefore, researchers have been striving to reduce the reinnervation time to salvage the target motor endplates and end organ muscle and the chronically denervated distal nerve pathways..$^{12,13}$

ABBREVIATIONS CMAP = compound muscle action potential; Dil = 1,1'-dioctadecyl-3,3,3',3'-tetramethylindocarbocyanine perchlorate; ETE = end-to-end; ETS = end-toside; NIC = neuroma in continuity; SETS = supercharge ETS.

SUBMITTED June 30, 2017. ACCEPTED December 4, 2017.

INCLUDE WHEN CITING Published online May 18, 2018; DOI: 10.3171/2017.12.JNS171570.

* M.N. and S.R. share first authorship of this work. 
End-to-side (ETS) and end-to-end (ETE) nerve transfers ${ }^{11,38}$ have been used to repair severe proximal nerve injuries. $5,9,10,36$ Over the last 2 decades, distal ETE nerve transfers have become a suitable and perhaps better alternative to primary repair and grafting for severe brachial plexus injuries. ${ }^{18,31}$ Nonetheless, in cases of incomplete Sunderland grade II-IV traumatic NIC injuries and severe compressive (such as ulnar neuropathy at the elbow) neuropathies, an ETE transfer would eliminate the possibility of recovery from the native, regenerating axons. ${ }^{34} \mathrm{As}$ an alternative therapeutic option in these situations, a transfer of an intact nerve into the side of an injured nerve could augment its function and keep the possibility of axon regrowth through the native nerve. This technique is termed supercharge ETS (SETS) transfer, also referred to as reverse ETS transfer ${ }^{19,20}$ (the original ETS transfer is a coaptation of an injured nerve to the side of the intact nerve). ${ }^{11,14,25}$

Several authors have demonstrated the utility of SETS for nerve injury in an array of experimental studies ${ }^{11,20,21}$ with a constellation of models ranging from an incomplete recovery model using an isograft to a transection with or without repair, none of which have aptly simulated an NIC injury. Moreover, a few clinical papers have shown promising results of using SETS transfer for enhancing intrinsic hand muscle function in proximal ulnar nerve injuries, yet the level of evidence is still weak..$^{4,7}$ In light of the current literature, no proven surgical options for the treatment of severe NIC exist, for which SETS may be even more applicable. This prompted us to assess the utility of SETS in an NIC experimental model ${ }^{2,3}$ in a way that emulates a clinical scenario. In this paper, the effectiveness of SETS transfer in NIC injuries and the degree of contribution from native and foreign neuronal pools toward enhanced neuronal regeneration were examined.

\section{Methods \\ Study Design}

This study was conducted in 2 phases. Phase I was designed to assess the effectiveness of distal SETS repair to enhance motor neuronal regeneration following NIC injuries in rodents. Phase II was designed to evaluate the level of contribution of native tibial and donor peroneal pools and examine the impact of NIC on SETS and vice versa. Adult male Lewis rats ( 7 weeks old, Charles River Laboratories International Inc.) were used in both phases of the experiment, under institutional animal care committee-approved guidelines (Fig. 1A).

\section{Phase I}

A total of 35 inbred Lewis rats were randomly assigned to one of 5 groups for right sciatic nerve surgeries. Under sterile conditions, the sciatic nerve was exposed between the sciatic notch and popliteal fossa, and neurolysis was performed to separate the tibial and peroneal nerves for at least $20 \mathrm{~mm}$. The peroneal nerve was cut at the popliteal fossa above the knee in all rats. In group 1 (treatment group, $\mathrm{n}=10)$, double NIC injuries ( $1 \mathrm{~mm}$ apart) were applied over the proximal tibial nerve., ${ }^{2,3}$ A traction spring scale was used to apply a $50 \mathrm{~g}$ traction force, perpendicular to the tibial nerve course, along with intense focused compression with a malleus nipper for 3 seconds over the most proximal tibial nerve. Immediately thereafter, SETS repair was done through an epineural window on the tibial nerve, $15 \mathrm{~mm}$ distal to the injury site and $5 \mathrm{~mm}$ proximal to tibial nerve trifurcation. The proximal stump of the peroneal nerve was coapted with the tibial nerve using 10-0 nylon sutures (Fig. 1B). The distal peroneal nerve stump was capped and then inverted away from the repair site. In group 2 (injury control, $\mathrm{n}=10$ ), NIC injury was done over the proximal tibial nerve, as in group 1 . The peroneal nerve was severed, and the proximal and distal stumps of this nerve were both capped (with a Silastic blind-ended tube of $0.51 \mathrm{~mm}$ inner diameter and $0.94 \mathrm{~mm}$ outer diameter) and inverted. In group 3 (positive control, $n=5$ ), the tibial nerve was not injured and only the peroneal nerve was transected, followed by capping of both stumps. In group 4 (SETS control, $n=5$ ), the proximal tibial nerve was transected and capped, followed by SETS repair as in group 1. In group 5 (negative control, $\mathrm{n}=5$ ), both tibial and peroneal nerves were transected followed by capping. In all rats, the muscle and skin were reapproximated using 4-0 Vicryl sutures and staples (MikRon autoclip $9 \mathrm{~mm}$ ).

Surgical procedures were performed under inhalation anesthesia (isoflurane, Pharmaceutical Partners of Canada Inc.) using standard microsurgical and aseptic techniques. Buprenorphine $(0.03 \mathrm{mg} / \mathrm{kg})$ subcutaneous injections followed by buprenorphine in flavored gelatin were used for peri- and postoperative analgesia. Animals were euthanized at study termination, under deep inhalation anesthesia with intraperitoneal Somnitol (Bimeda-MTC), followed by transcardial perfusion with saline and $2 \%$ paraformaldehyde.

\section{Outcome Measurements in Phase I \\ Electrophysiology Studies}

Electrophysiological assessment was performed utilizing compound muscle action potentials (CMAPs) performed under anesthesia at 2, 4, 6, 8, and 10 weeks. The nonoperated hindlimb was tested for control values. Stimulation of the sciatic nerve with single electrical pulses $(100-\mu$ sec duration and supramaximal intensity up to 10 $\mathrm{mA}$ ) was delivered using monopolar needles percutaneously placed at the sciatic notch, well proximal to the injury. CMAPs were recorded from the posterior compartment muscles (active at gastrocnemius and soleus muscles and reference at the ankle tendon).

\section{Behavioral Analysis}

Sensorimotor recovery was serially evaluated using the skilled ladder rung locomotion task, which is a reliable measure for detecting subtle sensorimotor changes in rats with sciatic nerve and branch injuries. ${ }^{3,23,24,30}$ All animals were trained to cross a horizontally placed ladder prior to surgical intervention. During testing, an irregular pattern of the rungs ( $1-3 \mathrm{~cm}$ apart) was changed from trial to trial to prevent learning effects. Three satisfactory runs were used for each rat and time point, consisting of the rat traveling across the beam uninterrupted at a constant velocity. Steps with the right hindlimbs were scored as a correct or an incorrect (total miss, deep or slight slip) step. A slip 


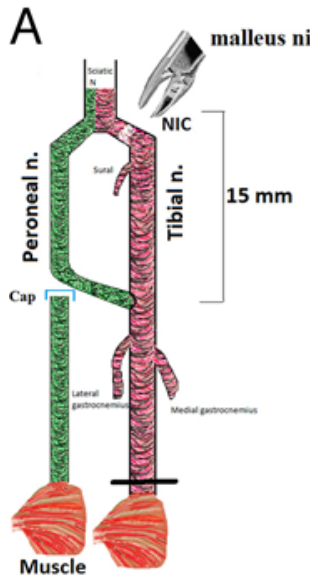

G1

Treatment group

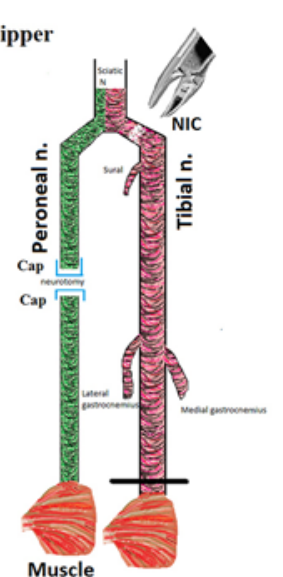

G2

Injury control

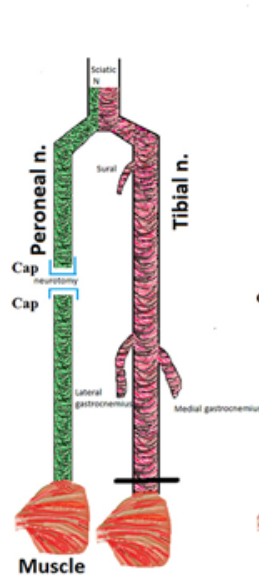

G3

Positive control

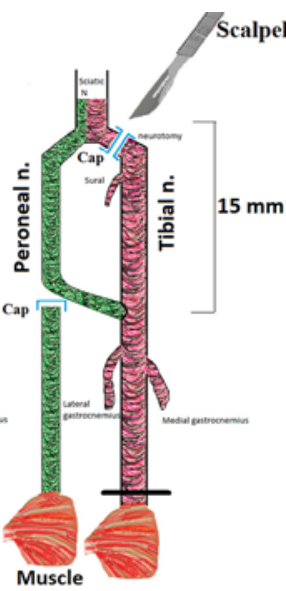

G4

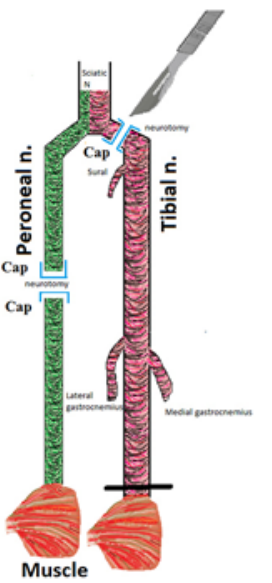

G5

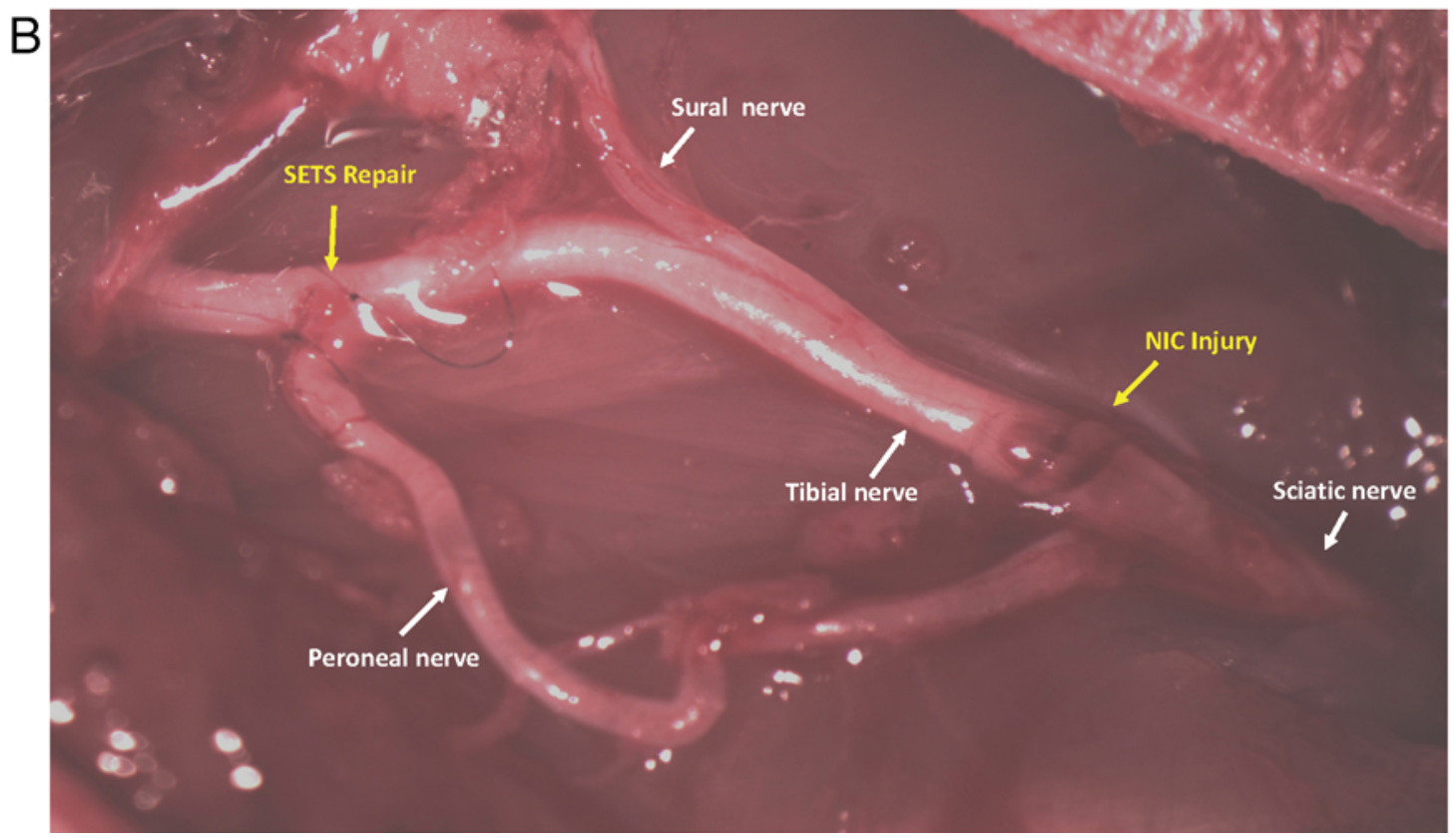

FIG. 1. A: Experimental groups. Group 1 (G1), treatment group, NIC with SETS. Group 2 (G2), injury control group, NIC without SETS. Group 3 (G3), positive controls. Group 4 (G4), SETS control group, transection with SETS. Group 5 (G5), negative control group. The tibial nerve was injured close to its origin, with NIC performed using a malleus nipper. SETS was performed from the peroneal nerve (green), through an epineural window $15 \mathrm{~mm}$ distal to the injured segment of the tibial nerve (pink). All stumps were capped following transection to prevent spurious regeneration. Distal black bars represent sections for histomorphometric analysis and the site for final introduction of the retrograde tracer. Nippers are used to create the NIC injuries, and the scalpel to create the neurotomies. B: SETS repair in the treatment group (G1). There is a double NIC injury in the proximal tibial nerve. SETS was performed $15 \mathrm{~mm}$ distal to the injured segment between the peroneal and tibial nerves using 10-0 sutures. Figure is available in color online only.

ratio $(\%)$ was calculated as the number of right hindlimb incorrect steps per total number of right hindlimb steps. Recordings were analyzed frame by frame by a trained blinded observer. Data were analyzed for baseline and weeks $2,4,6,8$, and 10 following surgery.

\section{Histomorphometric Analysis}

At week 10 during capping for retrograde labeling, a 3-mm segment of distal tibial nerve was harvested and fixed overnight in $2.5 \%$ glutaraldehyde buffered in cacodylate $(0.025 \mathrm{M})$. Washed samples were postfixed in osmium tetroxide (2\%) for 2 hours and then embedded in Epon. Semi-thin transverse sections (approximately $1 \mu \mathrm{m}$ ) were cut and stained with toluidine blue. Seven fields of view per section were photographed at high-power light microscopy ( $\times 400$, BX51, Olympus) for analysis. Images were digitized using a Wacom Intuos 3 digitizing tablet and analyzed using Image-Pro Plus software (Media 
Cybernetics) by an observer blinded to treatment groups. Parameters measured included the number of myelinated axons, percentage of neural tissue, nerve fiber diameter, and g-ratio, which measures the amount of myelination.

\section{Spinal Cord Motor Neuron Pool Retrograde Labeling}

At 10 weeks, retrograde labeling using fast blue (Polysciences Inc.) was performed by capping the proximal stump of the severed distal tibial nerve $(10 \mathrm{~mm}$ distal to the SETS) with small, prefilled silicone caps ( $2.5 \mathrm{~mm}$ long, $0.51 \mathrm{~mm}$ inner diameter). At 13 days postcapping, the rats were euthanized, and the lumbar spinal cord (T-12 to conus) was harvested, cryoprotected in paraformaldehyde and sucrose solution, and embedded in optimal cutting temperature compound for longitudinal (coronal) $45-\mu \mathrm{m}$ cryostat sectioning. All sections through the ventral gray horns were collected and mounted on glass slides. With the fluorescent microscope and appropriate filters, the total number of positively labeled fast blue cells was assessed by a blinded observer (the sum of all labeled neurons counted on all the slides of each spinal cord). Normally, the spinal cord has 2 discrete neuronal pools in the L-4 to L-6 segments contributed by the tibial and peroneal nerves..$^{33,35}$ The tibial pool is represented caudo-posteromedially, whereas the peroneal pool is found rostro-anterolaterally with a narrow area of overlap at the L-5 segment.

\section{Muscle Mass}

Prior to transcardiac perfusion, muscles innervated by the tibial nerve (gastrocnemius, soleus, and deep flexors of digits) were harvested from both the surgical and nonsurgical limbs and weighed using an analytical balance and are reported as a ratio (surgical/nonsurgical side).

\section{Phase II}

This phase was conducted on 20 rats by using both 1,1'-dioctadecyl-3,3,3',3'-tetramethylindocarbocyanine perchlorate (DiI) and fast blue for primary and secondary retrograde labeling, respectively., ${ }^{3,22,39}$ The sequential application of double retrograde labeling enables the evaluation of pre- and postoperative distribution of motor neurons in the same animal. The double-labeled cells would be helpful in identifying those native neurons that had reestablished connections with their target end organ. ${ }^{6,22,29}$

\section{Primary Retrograde Labeling}

All 20 rats initially underwent prelabeling with 2\% DiI (Molecular Probes) in $0.9 \% \mathrm{NaCl}$. Under aseptic precautions, the distal sciatic nerve was exposed along with its bifurcation into tibial and common peroneal nerves on the right side. The tibial nerve was dissected in the popliteal fossa until its trifurcation. The prelabeling was performed using the direct injection technique (Fig. 1A). The tip of a 30-gauge needle attached to a 10- $\mu$ l Hamilton syringe was introduced proximal to the origin of the gastrocnemius branches and pushed $1-3 \mathrm{~mm}$ in a proximal direction followed by injection of $5 \mu \mathrm{l}$ of $2 \%$ DiI. The injection site was wrapped with a sterile Gelfoam pledget to avoid spillage and dye contact with the peroneal nerve, which was $>1 \mathrm{~cm}$ away from the tibial nerve.

\section{Surgical Procedure}

After 2 weeks of prelabeling, the animals were assigned to one of the 3 groups: group $1(n=8)$, group $2(n=7)$, and group $4(n=5)$. In all animals, the previous operative incision was reexplored, and appropriate surgical experiments were performed (see phase I).

\section{Secondary Retrograde Labeling}

At 10 weeks postprocedure, retrograde labeling using fast blue was performed in a similar fashion as in phase I. Using the fluorescent microscope and appropriate filters, the total number of DiI, fast blue, and double-labeled cells was assessed by a blinded observer (the sum of all labeled neurons counted on all the slides of each spinal cord). Note that the secondary label (like in phase I) was applied in the distal tibial nerve close to the ankle and so would exclude a large portion of the native tibial motor neuron pool, which branches to muscles near the popliteal fossa.

\section{Statistical Analysis}

Data were analyzed with GraphPad Prism 4 software using 1-way ANOVA, the unpaired t-test with Welch correction, and post hoc Tukey's multiple comparison test to compare results between groups. Statistical significance was accepted at the level of $p<0.05$, with results presented as the mean \pm SEM.

\section{Results \\ Stages and Consequences}

In phase I, all rats but one survived. The rat that died was replaced with a new pretrained animal, which was added to the same group. At the end of the study, another animal suffered wound dehiscence. This animal survived with wound revision and could be used for assessment. After finishing phase I, phase II was conducted using 20 new animals. Although all animals survived the experiment, one rat from group 1 did not show any labeling in the spinal cord, presumably because of technical reasons. This animal was excluded from the study and 19 rats' results were analyzed.

\section{Phase I}

\section{Electrophysiological Studies}

All groups except groups 3 and 5 demonstrated progressive improvement in CMAPs at the final end point. Groups 3 and 5 had the highest and lowest CMAP amplitudes, respectively. The difference between the CMAP amplitudes of group 1 (NIC with SETS) and group 2 (NIC without SETS) was nonsignificant ( $p=0.12$; Fig. 2). Group 4 showed a pattern of improvement that was similar to those of groups 1 and 2, but the difference is not statistically significant.

\section{Behavioral Analysis}

Although group 3 showed the least slip ratio to start with, this ratio continued to slightly worsen (get higher) over the course of 10 weeks (Fig. 3). Moreover, by week 10 , groups 3 and 5 (the worst injury without SETS) showed a similar ratio of slips. Similar to group 3, group 5 did not 


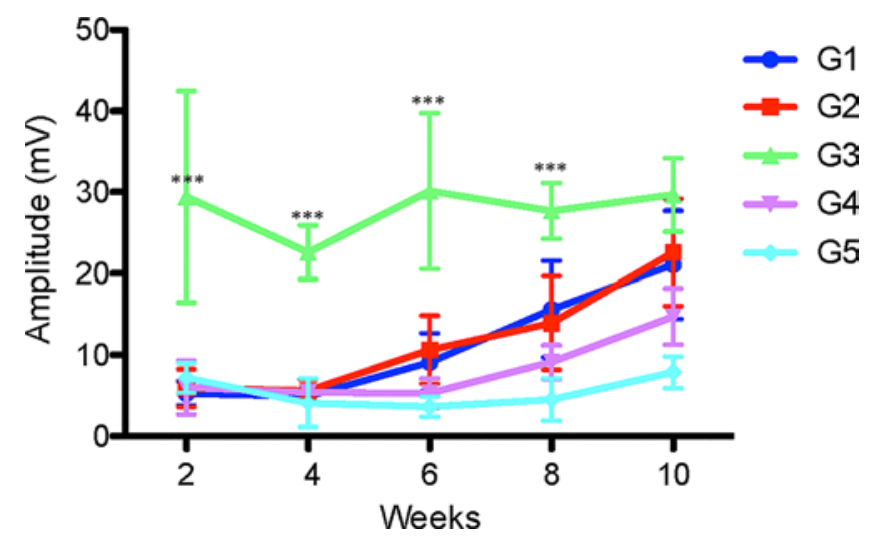

FIG. 2. Mean \pm SEM of CMAP amplitudes of the experimental groups. The sham tibial injury control group (group 3 ) exhibited a stable CMAP amplitude throughout the study that was significantly better $\left({ }^{* * *} p<0.05\right)$ than that of all other groups from the week 2 to week 8 assessments. The other groups all showed a progressive CMAP recovery at the final end point, except the negative control (group 5), which remained poor throughout. Note that there was no statistically significant difference between the other 3 groups. Figure is available in color online only.

show any improvement over the period of the experiment. On the other hand, although the slip ratios of groups 1 and 4 were higher than those in the other groups after week 4 , they were the only groups that exhibited improvement, although modest, over the observation period. Group 2 plateaued after the 4 th week. At week 10, there was no statistically significant difference between group 3 and group 1 or 4 .

\section{Retrograde Labeling}

All groups except group 5 had blue fluorescence in the ventral horn of the spinal cord. The intensity and amount of labeling were approximately $50 \%$ higher in group 1 (NIC with SETS) than in group 2 (NIC without SETS), and this difference was statistically significant $(\mathrm{p}<0.05)$. Rats in groups 1 and 4 had 2 pools (scattered and less

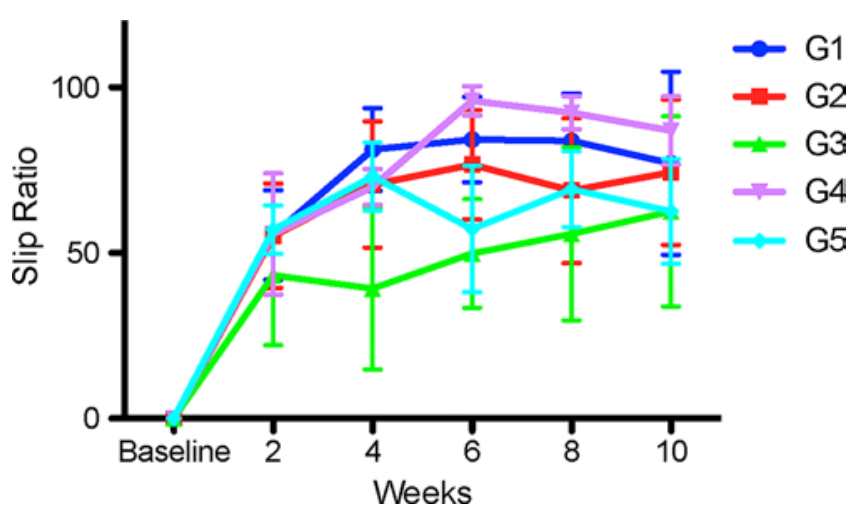

FIG. 3. Serial behavioral assessment by ladder rung tests with slip ratios plotted over time. The graph represents the mean slip ratios \pm SEM for each group. All groups demonstrate impaired locomotion throughout the study, with only modest improvement from week 4 to week 10 in the treatment group (group 1) and SETS control group (group 4), with no statistically significant difference between the other groups. Figure is available in color online only. dense), whereas those in groups 2 and 3 had only 1 pool (more compact and dense), which was contributed by the tibial nerve (Fig. 4). The additional rostral pool visualized in groups 1 and 4 was presumably from the peroneal nerve due to the SETS.

\section{Nerve Histomorphometry}

Cross sections of toluidine blue stained distal tibial nerve showed evidence of robust axonal regeneration in group 1 (Fig. 5). Histomorphometric analysis of the distal tibial nerve in group 3 showed a population of normal uninjured, well-myelinated axons with a larger mean fiber diameter $(6.48 \pm 0.59 \mu \mathrm{m})$. On the contrary, group 1 had numerous myelinated axons with a significantly smaller mean fiber diameter $(3.17 \pm 0.63 \mu \mathrm{m})$, which was not statistically different from group 2 or group 4 (Table 1). The myelinated axon counts were higher in group 1 at 945.60 $\pm 199.90 \mu \mathrm{m}$ than in group 2 at $733.83 \pm 229.72 \mu \mathrm{m}$, but the difference was not statistically significant $(\mathrm{p}=0.81)$.

\section{Wet Muscle Mass Analysis}

The mean weight ratios were distributed in the following order: group 3 had the highest weight ratio, followed by groups $2,1,4$, and 5 (Table 2). There was a noticeable positive effect of SETS on muscle mass when group 4 was compared with group 5.

\section{Phase II}

All 19 rats showed robust labeling of the ipsilateral ventral horn of the spinal cord (motor neuronal pool) with DiI $\left(\mathrm{D}_{0}\right.$ represents the tibial neuronal pool in Figs. 6 and 7). Fast blue labeling (Fig. 7B) reflects either the peroneal pool via SETS (groups 1 and 4, in this case an exclusively fast blue-labeled population [Fig. 6; $\mathrm{FB}_{0}$ in Fig. 7C]) or the regenerated tibial neuronal pool, including the doublelabeled population as in groups 1 and 2 (Fig. 6; FB in Fig. 7D).

The mean $\mathrm{D}_{0}$ counts were similar among the 3 groups ( $p=0.79$; Fig. 7A), representing the entire tibial motor neuron pool. The mean fast blue counts were significantly higher in group 1, followed by groups 2 and 4 (Fig. 7B). The mean $\mathrm{FB}_{0}$ counts were significantly higher in group 1 than in groups 4 and 2, and the counts in group 4 were slightly higher than those in group 2 (Fig. 7C). The mean $\mathrm{FB}_{+}$counts were significantly higher in group 2 than in group 1 (Fig. 7D).

\section{Discussion}

The SETS transfer technique was conceptualized to enhance the regenerative process without disturbing the native axons. Several studies have previously investigated this concept from different angles with promising results. ${ }^{11,19,20}$ In this study, we attempted to minimize the confounding factors and to address the previously raised criticisms around the previous work in this domain. Thus, we opted to use more control groups (including a true negative control), injury models emulating clinical scenarios, sufficient numbers of animals, blinded observers and analyzers, several outcome measures (and normal side controls as much as possible), robust locomotion function- 


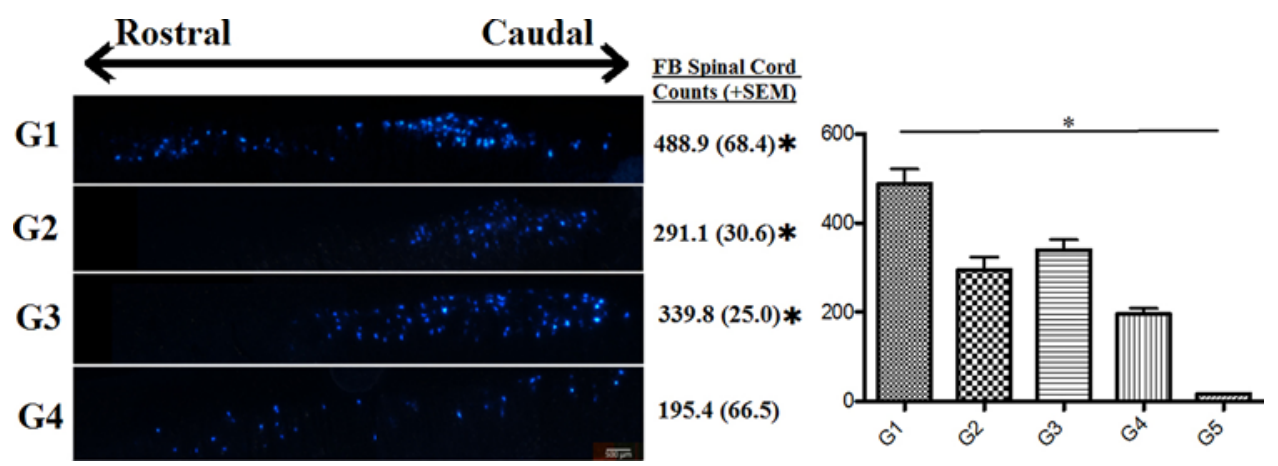

FIG. 4. Representative images and counts of retrograde-labeled motor neurons in the lumbar spinal cord sectioned in the longitudinal coronal plane following distal tibial nerve exposure to the fast blue (FB) tracer. Labeling in the treatment group (group 1) is dense and widespread, presumably reflecting contribution from both tibial (right side of image; more caudal and medial) and peroneal (left side of image; more rostral and lateral) neuronal pools. More restricted distribution of labeled cells in the caudal part of the spinal cord lumbar area reflects tibial motor neuron pools in groups 2 and 3 . Also, note the overall sparse, and less dense, labeling in the SETS control group (group 4), which represents the contribution from the peroneal nerve alone. Calibration bar is $500 \mu \mathrm{m}\left({ }^{*} p<0.05\right)$. Figure is available in color online only.

al tests compared with isolated muscle contraction tests (which were used in the majority of previous studies), and, for the first time, sequential double labeling in an attempt to measure the degree of contribution from each nerve and the effect of NIC on SETS and vice versa. Of note, we performed the coaptation immediately after the nerve injury, which is different from how these procedures are performed in clinical practice. We opted to conduct the experiments in this fashion for several technical reasons that aimed to avoid confounding factors. We strived to avoid any potential intervention that may influence the results. At the same time, we endeavored to be consistent in every step as much as practically possible. For instance, 1) the site of crush injury, 2) the double crush injury, 3) the force applied to create an injury to be as severe as grades III and IV, 4) the traction force on the nerve, 5) the site of the native nerve coaptation (15 $\mathrm{mm}$ from the crush), 6) the clean and clear-cut peroneal nerve SETS donor, and 7) the possibility of applying further stretch forces on the NIC (in the healing stage) during dissection cannot be equal during the identification steps of the second surgery to do SETS for all rats, and that by itself could be a confounding factor.

The results from the phase I outcome measures supported the regenerative effects from SETS transfer follow-
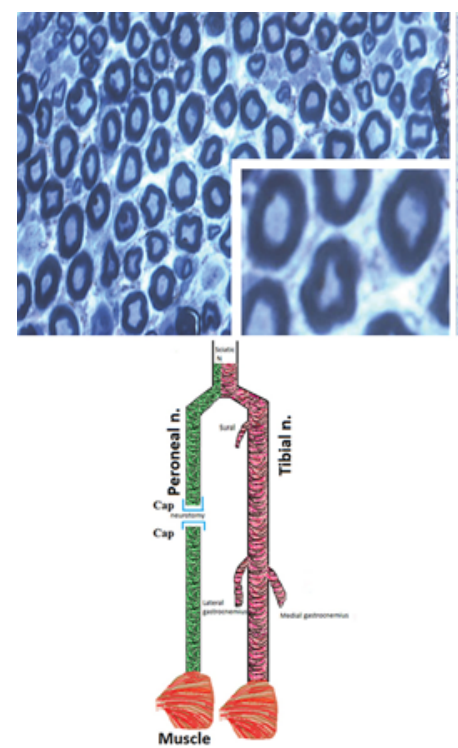

G3
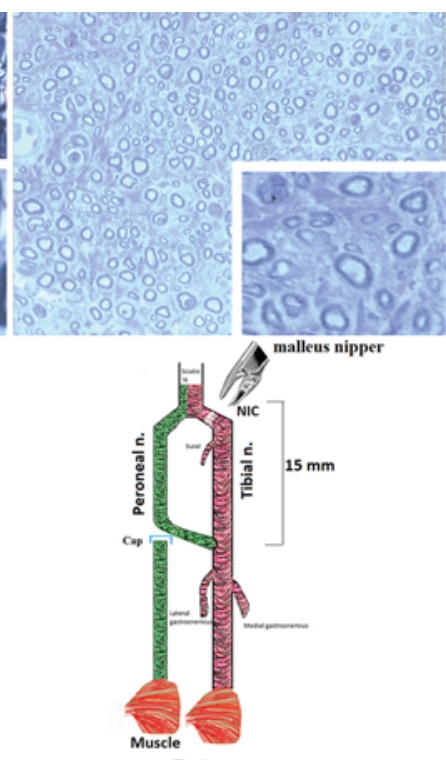

G1

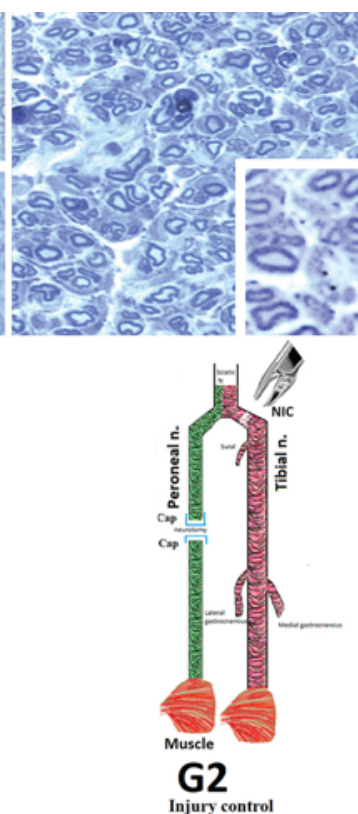

Injury control
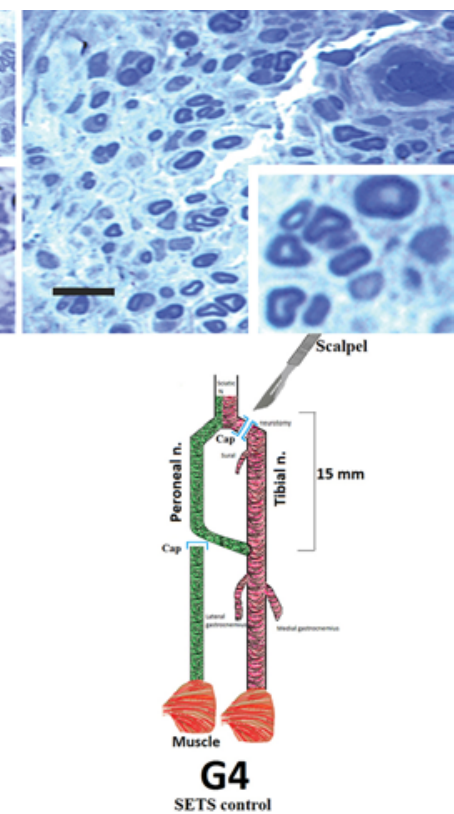

FIG. 5. Representative toluidine blue-stained cross sections of the distal tibial nerve of the various groups. Larger images correspond to $\times 40$ magnification, whereas the inset images are $\times 400$ magnification. The sham control group (group 3) contains a population of normally distributed, well-myelinated axons. All other groups show evidence of regenerated axons, which are somewhat smaller in caliber than the sham but well myelinated by week 10. Bar $=50 \mu \mathrm{m}$. Refer to Table 1 for quantitative summary of myelinated axon counts and other indices. Figure is available in color online only. 
TABLE 1. Histomorphometric indices at the final end point

\begin{tabular}{cccc}
\hline Group & Total Count (SEM) & Fiber Diameter (SEM) & g-Ratio (SEM) \\
\hline 1 & $945.6(199.9)$ & $3.17(0.63)^{*}$ & $0.66(0.88)$ \\
\hline 2 & $733.83(229.72)$ & $3.18(0.46)^{\star}$ & $0.69(0.82)$ \\
\hline 3 & $705.25(217.72)$ & $6.48(0.59)$ & $0.64(0.75)$ \\
\hline 4 & $744.8(307.35)$ & $2.46(0.33)^{*}$ & $0.61(0.68)$ \\
\hline
\end{tabular}

* Statistically significant from group $3(p<0.0001)$

ing proximal NIC injury. The rate of electrophysiological improvement in the groups in which SETS was used was obvious compared with the plateaued courses of groups 3 and 5. Moreover, group 4 (SETS alone) results were not significantly different from those of group 2. The larger number of fibers in groups 1 and 4 in histomorphometric analysis; the higher intensity, amount of labeling, and number of pools in group 1; and the noticeable positive effect of SETS on muscle mass in groups 1 and 4 all demonstrated the effect of SETS coaptation. Moreover, phase II experiments revealed that the majority of the contribution to reinnervation was from the transferred rather than the native motor neuron pool.

However, the improvement in functional testing was not as obvious as the other outcomes for several possible reasons. First, the skilled behavioral test used could have prevented functional improvements from being observed.
TABLE 2. Muscle weight data at the final end point

\begin{tabular}{cc}
\hline Group & Mean Wet Muscle Mass Ratio (SEM) \\
\hline 1 & $63.72(17.82)$ \\
\hline 2 & $70.08(13.3)$ \\
\hline 3 & $71.42(8.88)$ \\
\hline 4 & $60.95(16.22)$ \\
\hline 5 & $40.99(14.57)$ \\
\hline
\end{tabular}

Normalized muscle mass represents the ratio of the muscle mass (gastrocnemius, soleus, deep flexor of digits) of the surgical side to that of the nonsurgical side.

While we tested the spontaneous lower-limb function as a whole, others examined isolated muscle function. Isaacs et al. demonstrated encouraging results with SETS in a rat lower-limb model, measuring contractile forces of the extensor digitorum communis ${ }^{19}$ and the gastrocnemius mus$\mathrm{cle}^{20}$ in both of their experiments. Similarly, Farber et al. showed even more promising results of the SETS transfer when they measured gastrocnemius muscle force. ${ }^{11}$ Both groups of authors used nerve stimulation to evoke muscle contraction.

Second, and most importantly, our model of transfer resulted in innervation of antagonist muscles (nerve/muscle groups). Since we learned from phase II experiments that the majority of reinnervation is from the donor population,
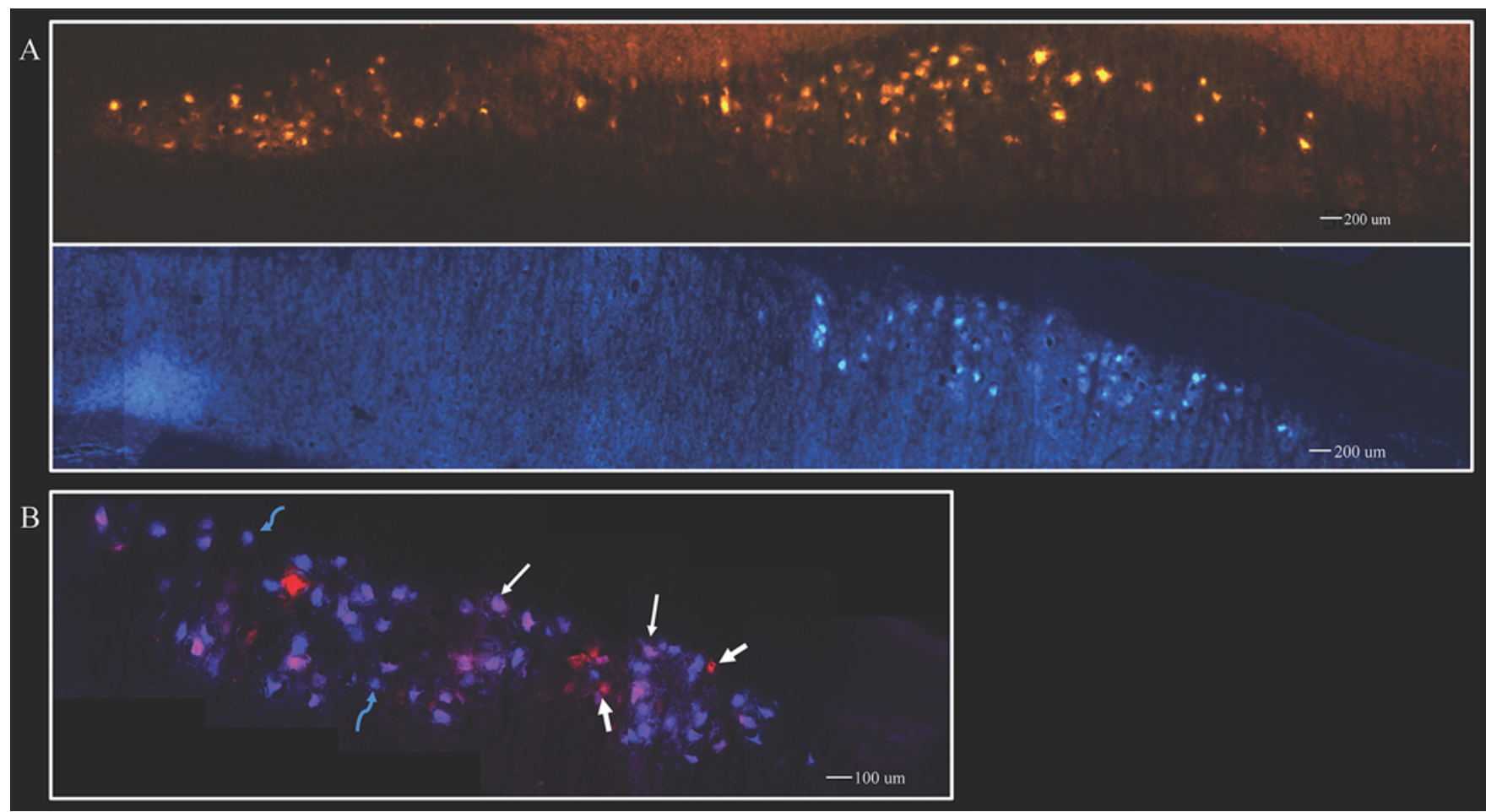

FIG. 6. Representative images of retrograde-labeled motor neurons in the lumbar spinal cord sectioned in the longitudinal coronal plane after initial distal tibial nerve exposure to Dil (red fluorescence) prior to surgery and fast blue tracer (blue label) postsurgery. A: Representative low-power micrograph from group 1 demonstrating the distribution of the tibial (Dil labeled) and peroneal pools (fast blue labeled). B: Group 2 gives rise to the highest amount of double-labeled neurons, which can be noted by the coloration of the neurons (double-labeled are light/dark purple, with examples denoted by arrows, while single-labeled neurons are clearly either red or blue). Figure is available in color online only. 


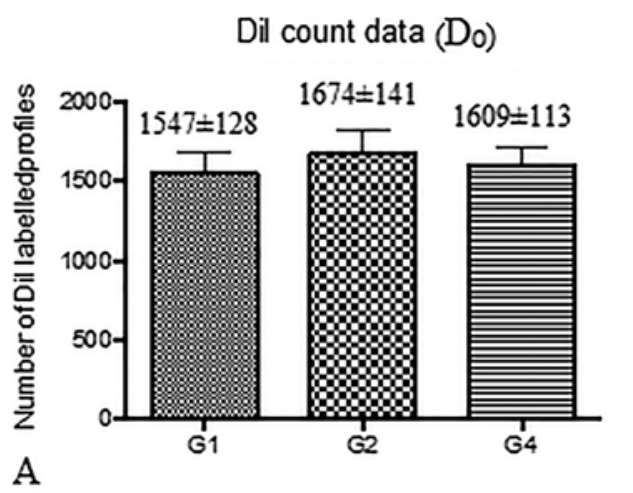

FB counts exclusive of doubles (FBo)

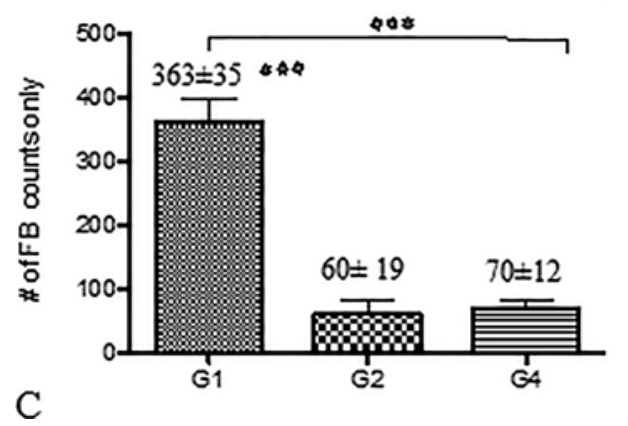

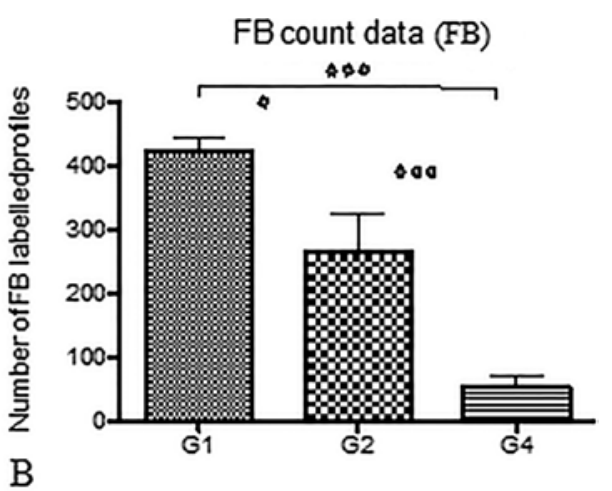

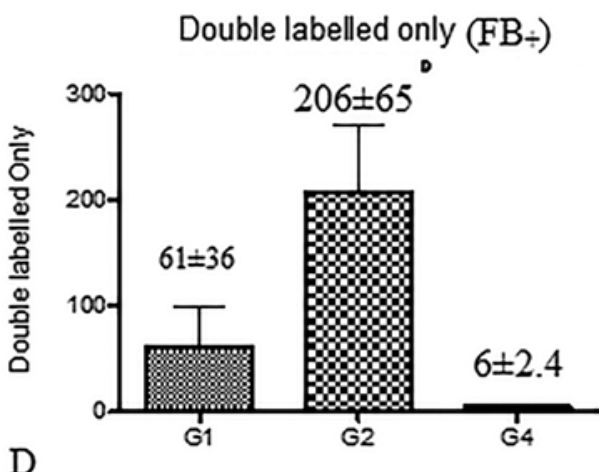

FIG. 7. Phase II counts (mean \pm SEM) from primary and secondary labeling of all experimental groups. A: The mean Dil labeling counts $\left(D_{0}\right)$ in groups 1,2 , and 4 represent the baseline number of motor neurons in the tibial pool. B: Total fast blue (FB) counts following secondary retrograde labeling from the distal tibial nerve at study termination. This is reflective of the collective labeling of all regenerated neurons and was found to be higher in group 1 than in group 2, suggestive of increased neuronal regeneration following SETS. The difference was statistically significant and is consistent with the findings in phase I. C: Exclusive FB (FB $)$ labeling count demonstrates that the group 1 labeling counts are higher than those in group 2 due to the presence of SETS. The group 4 counts are low, despite the presence of SETS, suggestive of better maturation of SETS in the presence of NIC compared with transection. The difference between groups 1 and 4 was statistically significant. D: The double-labeled count (FB $\left.{ }_{+}\right)$shows that the counts are lower in group 1 than in group 2, suggestive of decreased tibial neuronal regeneration across the NIC in the presence of SETS. ${ }^{*} p<0.05 ;{ }^{* * *} p<0.005$.

the innervation of tibial muscles from peroneal neurons may have resulted in poor integrated locomotor function. Indeed, classic studies by Gordon et al. reported poor outcomes in lower-limb models of the quadriceps involving whole nerve transfers innervating antagonistic muscles..$^{15,16}$ More recently, in their study of SETS involving peronealto-tibial transfer, Isaacs et al. observed that the preservation of both tibial and peroneal pathways yielded weaker muscle contraction because of less coordinated and simultaneous antagonistic muscle contraction. ${ }^{20}$ They finally concluded that these transfers achieved some functional recovery. Subtle recovery may occur over time, as demonstrated by selective nerve branch transfers between antagonistic nerve and muscle units. For instance, Kemp et al. transferred a lateral gastrocnemius nerve branch to the deep peroneal nerve in rats, compared it to direct deep peroneal repair, and examined their results using 2 functional systems: a skilled locomotion ladder rung system and ground reaction force determination. Although they found that the recovery of skilled locomotion function in the nerve transfer model was on par with that of animals who had direct innervation, the ground surface locomotion was significantly better in direct repair animals. ${ }^{23}$ In essence, while SETS worked to improve regeneration, we could not demonstrate an obvious functional effect, given the use of an antagonistic donor in a lower-extremity model. In this regard, Lutz et al. performed a comparative experimental study of ETS transfer between the median to ulnar nerve and the median to radial nerve. They found that the former had much better outcomes than the latter due to agonistic coaptation, underscoring the importance of using agonistic donors when considering SETS. ${ }^{27}$

Third, it is possible that transfer of the peroneal nerve (600 motor axons) to the tibial nerve (1000 motor axons) $)^{33}$ is not the ideal model to reflect obvious improvements. Intuitively, transfer of the tibial nerve to the peroneal side could provide a larger number of motor axons and could eventually show more obvious functional improvements. A fourth possibility is the relatively short duration of the experiment (the time from intervention to evaluation). It is noticeable from Fig. 3 that groups 1 and 4 are the only groups that showed continued improvement over the period of the study, while group 3 was getting worse and the other groups almost plateaued. It is possible that the longer period of observation may reveal further improvement in the SETS groups.

The significantly high fast blue counts in group 1 compared with group 2 confirm that the donor nerve (peroneal) 
provided the majority of the axons to the recipient nerve (distal tibial), and presumably these axons preferentially innervated the proximal tibial branch muscle targets. This finding supports the efficacy of this pattern of neurotization. Farber et al. showed similar results by using green fluorescent protein and a confocal imaging technique. ${ }^{11}$ The current work furthered the previous studies by showing the synergistic effect of this coaptation technique, which becomes clearer when the fast blue count of group 1 is compared with that of group 4 (Fig. 7). These findings reflect the positive impact of a proximal NIC on a distal SETS. An alternative explanation is that peroneal nerve axons may have preferentially regenerated proximally into the transected tibial nerve rather than distally and thus were not exposed to the secondary label in group 4 . The higher fast blue counts in group 2 than in group 4 can be interpreted by the larger number ${ }^{28,33}$ of motor neurons in the tibial pool than in the peroneal pool, albeit the group 4 fast blue count is promising by itself. The effectiveness of SETS is also obvious when analyzing the $\mathrm{FB}_{0}$ counts where the counts are higher in group 1 than in group 2 or 4. These findings represent the ability of SETS transfer to provide motor neurons to the target muscle.

Because of the proximity of SETS transfer to the denervated muscle, it is believed by some authors that SETS provides motor axons to the target endplate earlier than the regenerated axons via NIC (our model of NIC is $15 \mathrm{~mm}$ higher; Fig. 1A).$^{11}$ This may explain the markedly lower $\mathrm{FB}_{+}$count in group 1 compared with group 2 . The data suggest that peroneal nerve axons may have outcompeted axons arriving from the native tibial nerve, which were challenged to regenerate across the proximal NIC. These findings back the importance of SETS transfer, as motor endplate reinnervation in a timely fashion might secure significant muscle recovery even if it is supplied by less than half of the original number of motor units. ${ }^{17}$ To further understand the effect of SETS transfer on NIC, SETS could be eliminated for several weeks to allow the axons from the native nerve to establish themselves to the target muscle and then a tracer could be applied distally and the results compared with those of the group 2 double-labeled tibial pool.

The current study provides further evidence of SETS efficacy but also a cautionary note. On the positive side, we clearly demonstrate that SETS transfer (donor pathway) is the major source of regenerated axons to the recipient nerves. In addition, this work emphasized the trophic effect (synergy) of a proximal NIC on SETS when compared with transection. Although more than one group reported the positive impact of crush on this neurotization technique, the exact mechanism is not known. ${ }^{1,26}$ The cautionary note is related to the possible negative effect of SETS on regeneration across the NIC, but additional research is required to probe this further. Of note, although the majority of regenerated fibers come from the donor nerve, there are nerve fibers that regenerate from the native nerve through NIC that should not be simply neglected. In fact, investigating how to enhance regrowth through the NIC is an interesting therapeutic target in SETS transfer.

A limitation of our study is that we studied only immediate distal SETS transfer, whereas in clinical situations
SETS is usually performed in a delayed manner, so in the future it will be of interest to evaluate the motor neuron source in delayed SETS experimentally. Our research also emphasizes the importance of considering using the agonist muscle group at the time of SETS transfer compared with the antagonist group to enhance the functional outcome. Indeed, recent studies in the clinical field that report the possible effectiveness of SETS transfer are in the upper extremity and use agonistic donors. ${ }^{4,7}$

\section{Conclusions}

In experimental NIC injuries, the distal SETS transfer is the major source of regenerated axons to the recipient nerve. Caution therefore must be exercised in regard to the potential antagonistic effect of donor axons in choosing the donor nerve. The presence of a distal SETS coaptation may have a negative impact on neuronal regeneration across the NIC segment and requires further research.

\section{Acknowledgments}

This study was partially supported by the David Kline Research Award to Rajiv Midha from the Joint Disorders of Spine and Nerve Section of AANS/CNS.

\section{References}

1. Adelson PD, Bonaroti EA, Thompson TP, Tran M, Nystrom NA: End-to-side neurorrhaphies in a rodent model of peripheral nerve injury: a preliminary report of a novel technique. $\mathbf{J}$ Neurosurg 101 (1 Suppl):78-84, 2004

2. Alant JD, Kemp SW, Khu KJ, Kumar R, Webb AA, Midha R: Traumatic neuroma in continuity injury model in rodents. $\mathbf{J}$ Neurotrauma 29:1691-1703, 2012

3. Alant JD, Senjaya F, Ivanovic A, Forden J, Shakhbazau A, Midha R: The impact of motor axon misdirection and attrition on behavioral deficit following experimental nerve injuries. PLoS One 8:e82546, 2013

4. Baltzer H, Woo A, Oh C, Moran SL: Comparison of ulnar intrinsic function following supercharge end-to-side anterior interosseous-to-ulnar motor nerve transfer: a matched cohort study of proximal ulnar nerve injury patients. Plast Reconstr Surg 138:1264-1272, 2016

5. Beris AE, Lykissas MG: Experimental results in end-to-side neurorrhaphy. Int Rev Neurobiol 87:269-279, 2009

6. Bodine-Fowler SC, Meyer RS, Moskovitz A, Abrams R, Botte MJ: Inaccurate projection of rat soleus motoneurons: a comparison of nerve repair techniques. Muscle Nerve 20:29-37, 1997

7. Davidge KM, Yee A, Moore AM, Mackinnon SE: The supercharge end-to-side anterior interosseous-to-ulnar motor nerve transfer for restoring intrinsic function: clinical experience. Plast Reconstr Surg 136:344e-352e, 2015

8. de Ruiter GC, Malessy MJ, Alaid AO, Spinner RJ, Engelstad JK, Sorenson EJ, et al: Misdirection of regenerating motor axons after nerve injury and repair in the rat sciatic nerve model. Exp Neurol 211:339-350, 2008

9. Dellon AL, Ferreira MC, Williams EH, Rosson GD: Which end is up? Terminology for terminolateral (end-to-side) nerve repair: a review. J Reconstr Microsurg 26:295-301, 2010

10. Dvali LT, Myckatyn TM: End-to-side nerve repair: review of the literature and clinical indications. Hand Clin 24:455460, vii, 2008

11. Farber SJ, Glaus SW, Moore AM, Hunter DA, Mackinnon SE, Johnson PJ: Supercharge nerve transfer to enhance motor recovery: a laboratory study. J Hand Surg Am 38:466-477, 2013 
12. Fu SY, Gordon T: The cellular and molecular basis of peripheral nerve regeneration. Mol Neurobiol 14:67-116, 1997

13. Fu SY, Gordon T: Contributing factors to poor functional recovery after delayed nerve repair: prolonged denervation. $\mathbf{J}$ Neurosci 15:3886-3895, 1995

14. Fujiwara T, Matsuda K, Kubo T, Tomita K, Hattori R, Masuoka $\mathrm{T}$, et al: Axonal supercharging technique using reverse end-to-side neurorrhaphy in peripheral nerve repair: an experimental study in the rat model. J Neurosurg 107:821-829, 2007

15. Gordon T, Stein RB, Thomas CK: Innervation and function of hind-limb muscles in the cat after cross-union of the tibial and peroneal nerves. J Physiol 374:429-441, 1986

16. Gordon T, Stein RB, Thomas CK: Organization of motor units following cross-reinnervation of antagonistic muscles in the cat hind limb. J Physiol 374:443-456, 1986

17. Gordon T, Tyreman N, Raji MA: The basis for diminished functional recovery after delayed peripheral nerve repair. J Neurosci 31:5325-5334, 2011

18. Hems T: Nerve transfers for traumatic brachial plexus injury: advantages and problems. J Hand Microsurg 3:6-10, 2011

19. Isaacs J, Allen D, Chen LE, Nunley J II: Reverse end-to-side neurotization. J Reconstr Microsurg 21:43-50, 2005

20. Isaacs JE, Cheatham S, Gagnon EB, Razavi A, McDowell CL: Reverse end-to-side neurotization in a regenerating nerve. J Reconstr Microsurg 24:489-496, 2008

21. Kale SS, Glaus SW, Yee A, Nicoson MC, Hunter DA, Mackinnon SE, et al: Reverse end-to-side nerve transfer: from animal model to clinical use. J Hand Surg Am 36:1631-1639, 1639.e1-1639.e2, 2011

22. Katada A, Vos JD, Swelstad BB, Zealear DL: A sequential double labeling technique for studying changes in motoneuronal projections to muscle following nerve injury and reinnervation. J Neurosci Methods 155:20-27, 2006

23. Kemp SW, Alant J, Walsh SK, Webb AA, Midha R: Behavioural and anatomical analysis of selective tibial nerve branch transfer to the deep peroneal nerve in the rat. Eur J Neurosci 31:1074-1090, 2010

24. Kemp SW, Webb AA, Dhaliwal S, Syed S, Walsh SK, Midha $\mathrm{R}$ : Dose and duration of nerve growth factor (NGF) administration determine the extent of behavioral recovery following peripheral nerve injury in the rat. Exp Neurol 229:460-470, 2011

25. Kim CY, Kim YH: Supermicrosurgical reconstruction of large defects on ischemic extremities using supercharging techniques on latissimus dorsi perforator flaps. Plast Reconstr Surg 130:135-144, 2012

26. Kokkalis ZT, Soucacos PN, Terzis JK: Effect of donor nerve injury distal to an end-to-side neurorrhaphy model. J Reconstr Microsurg 25:295-306, 2009

27. Lutz BS, Chuang DC, Hsu JC, Ma SF, Wei FC: Selection of donor nerves-an important factor in end-to-side neurorrhaphy. Br J Plast Surg 53:149-154, 2000

28. Mackinnon SE, Dellon AL, O'Brien JP, Goldberg N, Hunter DA, Seiler WA IV, et al: Selection of optimal axon ratio for nerve regeneration. Ann Plast Surg 23:129-134, 1989
29. Madison RD, Archibald SJ, Brushart TM: Reinnervation accuracy of the rat femoral nerve by motor and sensory neurons. J Neurosci 16:5698-5703, 1996

30. Metz GA, Whishaw IQ: Cortical and subcortical lesions impair skilled walking in the ladder rung walking test: a new task to evaluate fore- and hindlimb stepping, placing, and co-ordination. J Neurosci Methods 115:169-179, 2002

31. Midha R: Nerve transfers for severe brachial plexus injuries: a review. Neurosurg Focus 16(5):E5, 2004

32. Noble J, Munro CA, Prasad VS, Midha R: Analysis of upper and lower extremity peripheral nerve injuries in a population of patients with multiple injuries. J Trauma 45:116-122, 1998

33. Schmalbruch H: Fiber composition of the rat sciatic nerve. Anat Rec 215:71-81, 1986

34. Sunderland S: A classification of peripheral nerve injuries producing loss of function. Brain 74:491-516, 1951

35. Swett JE, Wikholm RP, Blanks RH, Swett AL, Conley LC: Motoneurons of the rat sciatic nerve. Exp Neurol 93:227252,1986

36. Tos P, Artiaco S, Papalia I, Marcoccio I, Geuna S, Battiston B: Chapter 14: End-to-side nerve regeneration: from the laboratory bench to clinical applications. Int Rev Neurobiol 87:281-294, 2009

37. Valero-Cabré A, Navarro X: Functional impact of axonal misdirection after peripheral nerve injuries followed by graft or tube repair. J Neurotrauma 19:1475-1485, 2002

38. Viterbo F, Amr AH, Stipp EJ, Reis FJ: End-to-side neurorrhaphy: past, present, and future. Plast Reconstr Surg 124 (6 Suppl):e351-e358, 2009

39. Zhang L, McClellan AD: Fluorescent tracers as potential candidates for double labeling of descending brain neurons in larval lamprey. J Neurosci Methods 85:51-62, 1998

\section{Disclosures}

The authors report no conflict of interest concerning the materials or methods used in this study or the findings specified in this paper.

\section{Author Contributions}

Conception and design: Nadi, Midha. Acquisition of data: Nadi, Ramachandran, Islam, Forden, Guo. Analysis and interpretation of data: Nadi, Ramachandran, Midha. Drafting the article: Nadi, Ramachandran, Midha. Critically revising the article: Nadi, Midha. Reviewed submitted version of manuscript: Nadi, Ramachandran. Approved the final version of the manuscript on behalf of all authors: Nadi. Statistical analysis: Forden. Study supervision: Midha.

\section{Correspondence}

Mustafa Nadi: NSHA/Dalhousie University, Halifax, NS, Canada. mustafanadi@yahoo.com. 